\title{
GUIDELINES
}

\section{Updated S2K guidelines on the management of pemphigus vulgaris and foliaceus initiated by the european academy of dermatology and venereology (EADV)}

\author{
P. Joly, ${ }^{1, *}$ iD B. Horvath, ${ }^{2}$ A Patsatsi, ${ }^{3}$ S. Uzun, ${ }^{4}$ (D R. Bech, ${ }^{5}$ S. Beissert, ${ }^{6}$ R. Bergman, ${ }^{7}$ P. Bernard, ${ }^{8}$
} L. Borradori, ${ }^{9}$ iD M. Caproni, ${ }^{10}$ F. Caux,${ }^{11}$ G. Cianchini, ${ }^{12}$ (D) M. Daneshpazhooh, ${ }^{13}$ (D) D De, ${ }^{14}$ (ID M. Dmochowski, ${ }^{15}$ (D K. Drenovska, ${ }^{16}$ (D) J. Ehrchen, ${ }^{17}$ C. Feliciani, ${ }^{18}$ (D M. Goebeler, ${ }^{19}$ (D) R. Groves, ${ }^{20}$ (D) C. Guenther, ${ }^{21}$ (D) S. Hofmann, ${ }^{22}$ iD D. loannides, ${ }^{23}$ C. Kowalewski, ${ }^{24}$ R. Ludwig, ${ }^{25}$ Y.L. Lim, ${ }^{26}$ B. Marinovic, ${ }^{27}$ A.V. Marzano, ${ }^{28,29}$ (D) J.M. Mascaró Jr, ${ }^{30}$ (D) D. Mimouni, ${ }^{31}$ D.F. Murrell, ${ }^{32}$ (ID C. Pincelli, ${ }^{33}$ (D) C.P. Squarcioni, ${ }^{34}$ M. Sárdy, ${ }^{35}$ J. Setterfield, ${ }^{36}$ (iD E. Sprecher, ${ }^{37,38}$ (ID S. Vassileva, ${ }^{39}$ (D K. Wozniak, ${ }^{40}$ S. Yayli, ${ }^{41}$ (D) G. Zambruno, ${ }^{42}$ D. Zillikens, ${ }^{43}$ M. Hertl, ${ }^{44}$ (DD E. Schmidtt ${ }^{43}$ (iD)

${ }^{1}$ Department of Dermatology, Rouen University Hospital and INSERM U905, Centre de référence des maladies bulleuses autoimmunes, Normandie University, Rouen, France

${ }^{2}$ Department of Dermatology, University Medical Center Groningen, Center for Blistering Diseases, University of Groningen, Groningen, the Netherlands

${ }^{3}$ Autoimmune Bullous Diseases Unit, 2nd Dermatology Department, Papageorgiou General Hospital, Aristotle University School of Medicine, Thessaloniki, Greece

${ }^{4}$ Department of Dermatology, Akdeniz University Faculty of Medicine, Antalya, Turkey

${ }^{5}$ Department of Dermatology, Aarhus University Hospital, Aarhus, Denmark

${ }^{6}$ Department of Dermatology, University Hospital Carl Gustav Carus, Dresden, Germany

${ }^{7}$ Department of Dermatology, Rambam Health Care Campus and The Ruth and Bruce Rappaport, Faculty of Medicine, Haifa, Israel

${ }^{8}$ University of Reims-Champagne-Ardenne, Reims, France

${ }^{9}$ Department of Dermatology, University Hospital of Bern, University of Bern, Bern, Switzerland

${ }^{10}$ Department of Health Sciences, Section of Dermatology, USL Toscana Centro, Rare Diseases Unit, European Reference NetworkSkin Member, University of Florence, Florence, Italy

${ }^{11}$ Department of Dermatology and Referral Center for Autoimmune Bullous Diseases MALIBUL, Avicenne Hospital, AP-HP, University Paris 13, Bobigny, France

${ }^{12}$ Department of Dermatology, Cristo Re Hospital, Rome, Italy

${ }^{13}$ Autoimmune Bullous Diseases Research Center, Department of Dermatology, Razi Hospital, Tehran University of Medical Sciences, Tehran, Iran

${ }^{14}$ Department of Dermatology, Postgraduate Institute of Medical Education and Research, Chandigarh, India

${ }^{15}$ Autoimmune Blistering Dermatoses Section, Department of Dermatology, Poznan University of Medical Sciences, Poznan, Poland

${ }^{16}$ Department of Dermatology, Medical University, Sofia, Bulgaria

${ }^{17}$ Department of Dermatology, University of Münster, Münster, Germany

${ }^{18}$ Dermatology unit, Azienda ospedaliero -universitaria, Universita di Parma, Parma, Italy

${ }^{19}$ Department of Dermatology, Venereology and Allergology, University Hospital Würzburg, Würzburg, Germany

${ }^{20}$ St John's Institute of Dermatology, London, UK

${ }^{21}$ Department of Dermatology, University Hospital, Technical University Dresden, Dresden, Germany

${ }^{22}$ Department of Dermatology, Allergy and Dermatosurgery, Helios University Hospital Wuppertal, University Witten, Herdecke, Germany

${ }^{23} 1$ st Department of Dermatology-Venereology, Hospital of Skin and Venereal Diseases, Aristotle University Medical School,

Thessaloniki, Greece

${ }^{24}$ Department Dermatology and Immunodermatology, Medical University of Warsaw, Warsaw, Poland

${ }^{25}$ Department of Dermatology, University Hospital, Technical University Dresden, Dresden, Germany

${ }^{26}$ National Skin Centre, Singapore, Singapore

${ }^{27}$ Department of Dermatology and Venereology, University Hospital Centre Zagreb, School of Medicine, University of Zagreb, Zagreb, Croatia

${ }^{28}$ Dermatology Unit, Fondazione IRCCS Cà Granda Ospedale Maggiore Policlinico, Milan, Italy

${ }^{29}$ Department of Physiopathology and Transplantation, Università degli Studi di Milano, Milan, Italy

${ }^{30}$ Department of Dermatology, Hospital Clínic de Barcelona, Universitat de Barcelona, Barcelona, Spain

${ }^{31}$ Department of Dermatology, Rabin Medical Center, petha-Tikwa and Sackler School of Medicine, Tel Aviv University, Tel aviv, Israel

${ }^{32}$ Department of Dermatology, St George Hospital, University of NSW, Sydney, NSW, Australia

${ }^{33}$ DermoLab, Institute of Dermatology, University of Modena and Reggio Emilia, Modena, Italy

${ }^{34}$ Department of Dermatology and Referral Center for Autoimmune Bullous Diseases MALIBUL, Avicenne Hospital, AP-HP, University Paris 13, Bobigny, France

${ }^{35}$ Department of Dermatology, Venereology, and Dermatooncology, Semmelweis University, Budapest, Hungary 


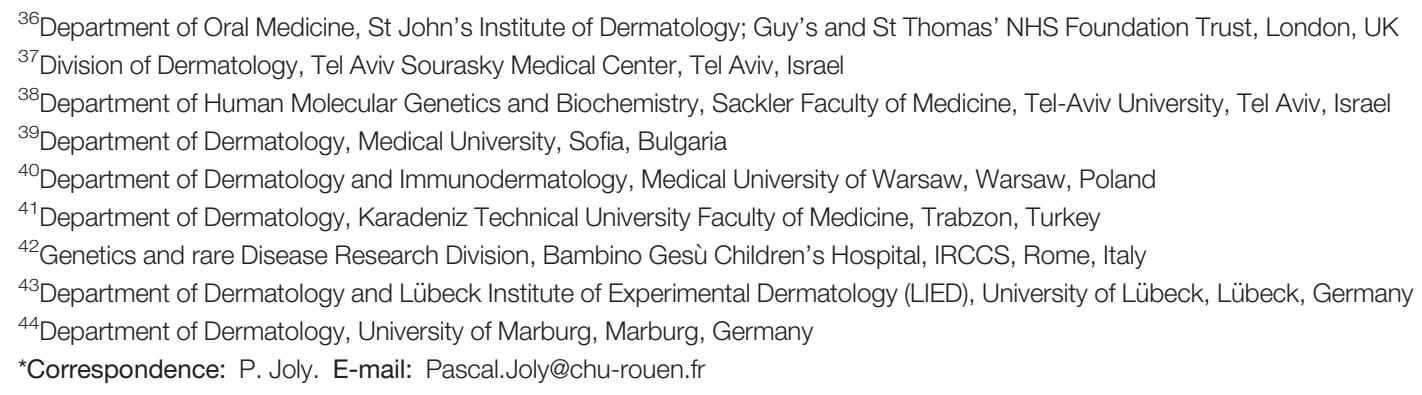

[Correction added on 28 August 2020, after first online publication: the name of author 'B. Horvath' has been corrected in this version.]

\section{Abstract}

Background Pemphigus encompasses a group of life-threatening autoimmune bullous diseases characterized by blisters and erosions of the mucous membranes and skin. Before the era of immunosuppressive treatment, pemphigus was almost always fatal. Due to its rarity, only few randomized controlled therapeutic trials are available. Recently, rituximab has been approved as first-line treatment for moderate and severe pemphigus vulgaris in Europe and the United States.

Objectives The Autoimmune blistering diseases Task Force of the European Academy of Dermatology and Venereology (EADV) has initiated a throughout update of the guideline for the management of patients with pemphigus.

Results The guidelines for the management of pemphigus were updated, and the degree of consent among all task force members was included. The final version of the guideline was consented by the European Dermatology Forum (EDF) and several patient organizations.

Received: 20 March 2020; Accepted: 29 May 2020

\section{Disclosures}

Pascal Joly is consultant for Roche, Amgen, Principia Biopharma, argenx, AstraZeneca and Thermo Fisher. Enno Schmidt is consultant for UCB, Incyte, argenx, Roche, Genetech, AstraZeneca, Admirx, Synthon, Imevax and Thermo Fisher. In addition, he received honoraria form Novartis, Biotest and Fresenius. He has research grants with UCB, Incyte, argenx, Admirx, Synthon and Biotest. Jan Ehrchen is consultant for Boehringer Ingelheim, Sobi and Novartis, and received speaker fees from Pfizer, Chugai, Actelion and Janssen. Matthias Goebeler is investigator in a clinical trial sponsored by argenx. Stefan Beissert received speaker fees and is consultant for Roche. Aikaterini Patsatsi is consultant for Principia Biopharma, Janssen, Leo, Novartis, AbbVie, Lilly, UCB, Genesis Pharma - Greece. Detlef Zillikens has obtained support for research and development work, lecturing and consulting from AbbVie, argenx, Biotest, Euroimmun, Fresenius, Janssen and UCB Pharma within the last 3 years. Michael Hertl received unrestricted grants from Topas Therapeutics, Hamburg, Germany, argenx, Brussels, Gent, Janssen Cilag, Brussels and Novartis. Giovanna Zambruno is member of the IDMC for a trial on pemphigus (ClinicalTrials.gov Identifier: NCT03334058) sponsored by arGEN-X BVBA and Zwijnaarde, Belgium. Soner Uzun is consultant for Roche. Savas Yayli is investigator for Roche and Principia Biopharma. Miklós Sárdy is consultant for Janssen, Novartis, Leo Pharma, Egis, AbbVie and Sanofi. José M. Mascaró Jr. has received speaker fees from Clover Soluciones Globales de Marketing, Ferrer Internacional, LEO Pharma Spain, Loki \& Dimas, and M.S.D. de Espana S.A. Carlo Pincelli is consultant for PinCell srl and Mylan. Dimitrios loannides is investigator in clinical trials for pemphigus sponsored by Principia and GlaxoSmithKline. He is consultant and investigator in trials for other skin diseases for AbbVie, Genesis, Janssen, Lilly, Leo, Novartis and UCB. Frédéric Caux is consultant for Principia Biopharma and is investigator in clinical trials sponsored by Principia Biopharma and Roche. Dedee F Murrell is consultant for Roche, Principia Bio, Lilly, GSK, Novartis and Sanofi. Marian Dmochowski, Claudia Guenther, Philippe Bernard, Jane Setterfield, Marzia Caproni, De Dipankar, Katarzyna Wozniak, Angelo Valerio Marzano, Daniel Mimouni, Silke Hofmann, Rikke Bech, Yen Loo Lim, Cezary Kowalewski, Kossara Drenovska, Maryam Daneshpazhooh, Giuseppe Cianchini, Claudia Guenther, Reuven Bergman, Branka Marinovic, Catherine Prost Squarcioni, Claudio Feliciani, Richard Groves and Snejina Vassileva have no conflict of interest. 


\section{Funding statement}

The guideline update was partly supported by the European Network for Rare skin Disorders (ERN) and the European Academy of Dermatology and Venereology (EADV).

\section{Introduction}

Pemphigus encompasses a group of life-threatening autoimmune bullous diseases characterized by flaccid blisters and erosions of the mucous membranes and/or skin. ${ }^{1-5}$ The severity of the disease is based on its progressive course which is accompanied by an increased body catabolism with loss of body fluids and proteins and secondary bacterial and viral infections which may lead to sepsis and cardiac failure. Before the advent of systemic corticosteroids, pemphigus was almost always fatal within two years after making the diagnosis. Pathophysiologically, the underlying intraepithelial blister formation is caused by IgG autoantibodies against the desmosomal adhesion proteins, desmoglein 3 (Dsg 3) and/or desmoglein 1 (Dsg1), on the cell surface of epidermal keratinocytes. ${ }^{5,6}$ Pemphigus is rare, and its incidence has been estimated to about 2 new patients per 1 million inhabitants per year in Central Europe. ${ }^{7,8}$ Two main clinical variants are known, pemphigus vulgaris (PV) and pemphigus foliaceus (PF). The pathogenic role of anti-desmoglein 1 and 3 IgG has been clearly established since the injection of patients' sera or affinity-purified IgG from pemphigus sera into neonatal mice reproduces clinically and immunopathologically the cardinal symptoms of pemphigus within $24 \mathrm{~h}^{1,5}$. In most patients, disease activity is closely correlated with serum levels of desmoglein-reactive autoantibodies. ${ }^{9,10}$ Due to its rarity, only few randomized controlled trials (RCT) are available in pemphigus, most of them limited by the low numbers of patients enrolled and the lack of statistically significant differences between the study groups. A few studies compared different doses of prednisone/prednisolone, i.v. corticosteroid pulses versus placebo, azathioprine versus mycophenolate mofetil, and the use of adjuvant treatment with methotrexate, cyclosporine, cyclophosphamide or high-dose intravenous immunoglobulins. ${ }^{11,12}$. The combination of systemic corticosteroids (prednisone/prednisolone, $1.0-1.5 \mathrm{mg} / \mathrm{kg} /$ day) and potentially corticosteroid-sparing immunosuppressive drugs, mostly azathioprine and mycophenolate mofetil, was regarded as standard first-line therapy by most clinicians. ${ }^{13}$

Over the past 15 years, more than 1,000 pemphigus patients treated with rituximab (monoclonal antibody against the CD20 molecule on B lymphocytes) have been reported in the literature. ${ }^{14}$ Rituximab has first been used to treat severe forms (recalcitrant or relapsing types) and later as first-line treatment reaching complete remissions in $80-90 \%$ of patients. ${ }^{15-18}$ Recently, a randomized controlled trial with newly diagnosed patients with PV and PF showed that $89 \%$ of patients assigned to the rituximab group achieved complete remission off therapy versus $34 \%$ of patients assigned to treatment with prednisone alone. ${ }^{18}$ Additionally, prednisone could be stopped after only 6 months of treatment in around $70 \%$ of patients initially treated with rituximab, leading to a twofold decrease in the number of severe treatment side-effects. ${ }^{18}$

For this reason, the Autoimmune blistering diseases task force of the European Academy of Dermatology and Venereology (EADV) has initiated a thorough update of the previous guidelines for the management of patients with pemphigus. ${ }^{13}$

\section{Methodology of guideline preparation}

To facilitate this process, a writing group, i.e. $\mathrm{PJ}, \mathrm{BH}, \mathrm{AP}, \mathrm{SU}$, $\mathrm{DZ}, \mathrm{MH}$ and ES, appointed by the Autoimmune blistering diseases task force of the EADV wrote the first version of the updated guidelines following two telephone conferences. Thereafter, all 62 members of the EADV Autoimmune blistering diseases task force (notation group) were invited to assign scores (ranging from 0 to 5 according to the increasing degree of consensus) to each of the recommendations statements using the syntax shown below. This process identified the statements of major agreement or disagreement. Indicated major statements were then voted upon, and the degree of consensus was indicated for all statements. Based on the marks of the notation group, the writing group then prepared a second and a third version of the guidelines, until each of the statements was given a mark $>4$ by the voting group. In this process, a consensus meeting was held during the annual meeting of the EADV in Madrid, Spain, September 2019. The manuscript was then reviewed by the different European patient organizations. The revised version of the guideline was finally passed to the European Dermatology Forum (EDF) for final consensus.

\begin{tabular}{ll}
\hline Grade of recommendation & Syntax \\
\hline Strong recommendation & Is recommended \\
Recommendation & May be recommended \\
Recommendation pending & May be considered \\
Negative recommendation & Is not recommended \\
\hline
\end{tabular}

\section{Initial evaluation of pemphigus}

The initial clinical examination should seek basic evidence for the diagnosis of pemphigus, as well as screening for co-morbidities.

\section{Major objectives}

To confirm the clinical diagnosis of pemphigus.

To search for risk factors, severity factors and potential comorbidities based on history and initial clinical evaluation. 
To specify the type of initial involvement (skin, mucosa) and its extent.

To evaluate the prognosis depending on the age of the patient, and general condition.

To measure extent and distribution of the lesions by Autoimmune Bullous Skin Intensity and Severity Score (ABSIS) or Pemphigus Disease and Area Index (PDAI).

To measure the impact on patients' quality of life using dermatology-specific score (DLQI) and tools specific for autoimmune blistering diseases (ABQOL, TABQOL). ${ }^{19,20}$

To start treatment expecting to achieve disease control and complete remission as soon as possible.

\section{Professions involved}

The treatment plan for patients with pemphigus is the responsibility of an experienced dermatologist, usually a hospital-based dermatologist in a tertiary referral centre, a specialized centre or a member of a network.

Other health professionals who may have supportive functions are as follows:

- The consultant dermatologist in general practice.

- The patient's general practitioner.

- All other specialists whose expertise is necessary, based on the patient's general clinical condition, co-morbidities, such as internists, cardiologists, stomatologists, ophthalmologists, otorhinolaryngologists, gastroenterologists, gynaecologists, urologists, proctologists, rheumatologists, oncologists and psychologists.

- Health nurses in selected cases in which home care is required and applicable, e.g. elderly or disabled patients with residual mucosal or skin lesions following hospitalization

- Dietician, physiotherapist

- Nurse specialist/practitioner

It is recommended that a dermatologist experienced in the management of pemphigus is involved in setting up the initial treatment plan.

It is recommended that other health professionals (as listed above) are involved in the management of the patient according to the sites of involvement, disease severity, comorbidities and complications.

\section{Clinical examination}

Medical history It is recommended to document the following points:

Specify the time of first onset of symptoms.

Specify functional symptoms, i.e. pain, pruritus, intensity of dysphagia, ocular and ENT symptoms, dysuria, anogenital problems and weight loss.

Record haematological, oncologic, endocrine, cardiovascular and infectious medical history to search for risk factors of oral corticosteroid treatment and evolving complications of immunosuppressive therapy.

Evaluate anticipated pregnancy, actively practiced contraception (especially if immunosuppressive treatment is being considered).

Ask for recent drug intake which may potentially induce pemphigus, such as angiotensin-converting enzyme (ACE) inhibitors, angiotensin receptor blockers, beta-blockers, cephalosporins, phenylbutazone, pyritinol, thiopronine and rifampicin. D-penicillamine, which was classically involved in drug-induced pemphigus, is almost no longer used. It may be considered whether to discontinue ACE inhibitors, angiotensin receptor blockers, beta-blockers, cephalosporins, phenylbutazone, pyritinol and thiopronine in the case of a clear temporal association between the occurrence of pemphigus lesions and the drug intake.

Evaluation of the impact on quality of life using validated questionnaires, e.g. DLQI, and/or the ABQOL and TABQOL questionnaires specific for autoimmune bullous diseases may be considered (4.2 \pm 1.2$)$.

Check for updated vaccination. Inquire about a possible trip in a country in which vaccination with a live vaccine is required (to perform this vaccination before using immunosuppressive treatment.)

\section{Physical examination}

General. It is recommended to assess the following aspects:

The extent of skin and mucous membrane lesions, the degree of mucosal damage and functional impairment (dysphagia, dysphonia, weight loss, impairment of vision, dyspareunia). It is recommended to quantify the extent of skin and mucosal lesion using one of the two validated scoring systems: the Pemphigus Disease Area Index (PDAI) or the Autoimmune Bullous Skin Intensity and Severity Score (ABSIS) $(4.5 \pm 1.0) .^{21,22}$

The patient's general condition and co-morbidities are as follows:

\section{Bodyweight.}

Arterial blood pressure.

General condition, co-morbidities (neoplastic, cardiovascular, musculoskeletal, diabetes, etc.).

The majority of authors do not recommend performing the classical Nikolsky's sign, considering that there are other tools to measure disease activity, and that it is not necessary to induce new lesions in patients who already have skin erosions.

Pemphigus vulgaris $(P V)$. Usually begins with oral mucosal lesions: buccal and/or gingival painful, persisting erosions which interfere with eating. Less common are non-cicatricial ocular lesions, and nasal, laryngeal, oesophageal, genital and anal erosions are also possible.

Cutaneous involvement (which may appear several weeks or months after the first appearance of mucosal lesions) presents 
with flaccid bullae with clear content, developing on non-erythematous skin quickly transforming into postbullous erosions.

The lesions may be localized or generalized and predominate at seborrhoeic areas (chest, face, scalp, interscapular region) and mechanically stressed regions as well as on the extremities.

The disease is usually not associated with major pruritus.

Fingernail involvement is possible.

Pemphigus foliaceus (PF). Cutaneous involvement: transient, flaccid bullae or puff pastry-like exfoliation transforming into crusty erosions in seborrhoeic skin areas (chest, scalp, face, interscapular region).

More extensive cutaneous involvement in sporadic and endemic pemphigus foliaceus ('Fogo Selvagem', Brazilian pemphigus, Tunisian pemphigus). No mucosal involvement.

Paraneoplastic pemphigus (PNP). To be suspected in the context of concomitant malignancy, particularly non-Hodgkin's lymphoma, chronic lymphocytic leukaemia, thymoma or Castleman's disease. In not all cases, the underlying malignancy has been diagnosed at the time of diagnosis.

Moreover, the symptoms of PNP can precede the malignancy.

Mucosal involvement: initially limited cheilitis and/or ulcerative stomatitis, persisting painful erosions which lead to severe dysphagia. Cicatricial conjunctivitis, keratitis and genital involvement are common. Pharyngeal involvement is possible; the nasal cavity and oesophagus can also be affected leading to phagodynia.

Cutaneous polymorphic lesions with symptoms resembling mild lichen planus-like to graft-versus-host disease-like, erythema multiforme-like, bullous pemphigoid-like or pemphigus vulgaris-like eruption. Palmar involvement is common.

Pulmonary involvement (alveolitis, bronchiolitis obliterans, pulmonary fibrosis) is a characteristic and life-threatening complication.

\section{Diagnosis}

\section{The diagnosis of pemphigus is based on four criteria}

1 Clinical presentation (see 2.3)

2 Histopathology

3 Direct immunofluorescence (DIF) examination of a perilesional skin or mucosal biopsy

4 Serological detection of autoantibodies against epithelial cell surface by indirect immunofluorescence (IIF) and/or enzymelinked immunosorbent assay (ELISA Dsg1 and Dsg3).

It is recommended to perform the following investigations for the diagnosis:

- A lesional biopsy for histopathology

- A perilesional biopsy for DIF examination

- Serology

Histopathology Preferentially, a 3- to 5-mm punch biopsy of a recent $(<24 \mathrm{~h})$ vesicle or $1 / 3$ of the peripheral portion of a blister and $2 / 3$ perilesional skin (placed in $4 \%$ formaldehyde solution) should be taken for routine histopathological analysis: intraepidermal suprabasal acantholysis in PV or acantholysis at the granular layer in PF. Epidermal acantholysis, suprabasal cleft formation, dyskeratotic keratinocytes, vacuolar change of the basilar epidermis and epidermal exocytosis of inflammatory cells (PNP).

Direct immunofluorescence microscopy Skin biopsy of perilesional skin or mucosa (up to $1 \mathrm{~cm}$ from a recent lesion), put into a cryotube for transportation in a cylinder of liquid nitrogen, or in saline (delivery $<36 \mathrm{~h}$ ) or Michel's fixative for DIF analysis: IgG and/or C3 deposits at the surface of epidermal/epithelial keratinocytes.

The epithelial cell surface staining for in vivo IgG deposition is normally granular or linear as observed by DIF examination.

Epithelial cell surface deposits can sometimes be associated with linear deposits of IgG or C3 along the dermal-epidermal junction, suggestive of PNP, or pemphigus erythematosus, or the coexistence of pemphigus and pemphigoid.

In specialized laboratories, plucked hairs can be utilized for DIF for the diagnosis of pemphigus.

\section{Immune serological tests}

Indirect immunofluorescence microscopy.

- IIF examination of patient' serum on monkey oesophagus or human skin to search for autoantibodies against surface proteins of epidermal keratinocytes. The smooth and reticular staining pattern is also referred to as 'chicken wire', 'honeycomb' or 'fishnet-like'.

- IIF microscopy based on cells that recombinantly express Dsg 1 and Dsg 3 on the cell surface (Biochip; Euroimmun).

In case of atypical presentation, suspected PNP, or the suspicion of an unrelated autoimmune bullous disorder, additional immunopathological tests may be performed, such as IIF microscopy on rat bladder and/or immunoblot/immunoprecipitation.

ELISA Detection of anti-Dsg1 (PF/mucocutaneous PV) and/or anti-Dsg3 IgG autoantibodies (Abs) (mucosal PV) by ELISA (Table 1).

The detection of anti-Dsg IgG autoantibodies by ELISA is positive in more than $95 \%$ of cases.

In general, ELISA values correlate with the extent and/or activity of disease (see remark above and prognostic value for relapse, helping to guide treatment).

Table 1 Commercially available ELISAs

\begin{tabular}{ll}
\hline Desmoglein 1 & MBL, Euroimmun \\
Desmoglein 3 & MBL, Euroimmun \\
Envoplakin $(210 \mathrm{kDa})$ & Euroimmun \\
\hline
\end{tabular}


It is recommended to make the diagnosis of PV/PF on the following findings:

Compatible clinical picture plus positive DIF examination

If DIF examination is negative, it is recommended to repeat the biopsy for DIF microscopy.

If a new biopsy is not available, or still negative, it is recommended to make the diagnosis of pemphigus in patients with:

- Compatible clinical picture plus corresponding typical histological picture of acantholysis plus significant positive IIF on monkey oesophagus: $(4.2 \pm 1.3)$. In this particular case, the presence of a typical histological picture of acantholysis is absolutely necessary to exclude an erythema multiforme major.

- Compatible clinical picture and histopathology plus serum reactivity against recombinant Dsg 1 and/or 3 by ELISA or IIF on cells that express recombinant Dsg1/Dsg3 $(4.7 \pm 0.8)$.

\section{Diagnosis of paraneoplastic pemphigus}

- In case of atypical clinical features (i.e. progressive stomatitis, lichenoid or erythema multiforme-like skin lesions), the diagnosis of PNP must be considered.

- Lesional histopathology frequently shows dyskeratosis and a lichenoid interface dermatitis in addition to acantholysis.

- DIF examination may reveal linear staining of IgG/C3 at the dermal-epidermal junction in addition to intercellular staining of the epithelium.

- IIF examination of serum on rat bladder shows cell surface staining of epithelial cells, corresponding to reactivity against plakin proteins.

- Immunoblot with epidermal extracts shows IgG against envoplakin $(210 \mathrm{kDa})$ and periplakin $(190 \mathrm{kDa})$, Dsg3 (130 kDa), and more rarely Dsg1 (160 kDa). Anti-desmocollin Abs can be occasionally detected, usually in association with anti-envoplakin Abs (Table 1) (4.6 \pm 1.2). Alpha-2-macroglobulinlike-1 (A2ML-1, $170 \mathrm{kDa}$ ) can only be detected under nondenaturing condition-non-reducing condition.

- Immunoblot with extracts from cultured keratinocytes reveals IgG against envoplakin $(210 \mathrm{kDa})$, periplakin (190 kDa) and desmoplakin I/II (210 and $250 \mathrm{kDa})$.

- Immunoprecipitation with keratinocyte extracts is not performed in clinical practice. It can reveal IgG autoantibodies against envoplakin $(210 \mathrm{kDa})$ and periplakin $(190 \mathrm{kDa})$, Dsg3 (130 kDa), Dsg1 (160 kDa), desmocollins, desmoplakins I and II, BP230/BPAG1, plectin (500 kDa) and alpha-2-macroglobulin-like-1 (A2ML-1, $170 \mathrm{kDa})$ $(4.6 \pm 1.5)$.

Work-up before corticosteroid or immunosuppressive therapy

It is recommended to perform the following investigations before treatment is initiated: $(4.8 \pm 0.9)$
Complete blood count;

Creatinine, blood electrolytes;

Transaminases, gamma GT, alkaline phosphatase;

Total serum protein, albumin;

Fasting serum glucose;

Hepatitis B, C and HIV;

Serum IgA (in case of intravenous immunoglobulin (IVIG) treatment);

G6PD activity, bilirubin and reticulocytes (in case of treatment with dapsone)

Thiopurine methyltransferase (TPMT) activity (in case of treatment with azathioprine)

Ocular examination to exclude glaucoma and cataract

It may be recommended to perform the following investigations before treatment is initiated: $(4.8 \pm 1.2)$

Chest X-ray (in case of elevated risk of tuberculosis)

Quantiferon test or PPD (in case of elevated risk of tuberculosis).

Abdominal sonography

ß HCG to exclude pregnancy in females of childbearing age;

Osteodensitometry prior to glucocorticoid treatment;

\section{Therapeutic management}

\section{Objectives}

The primary objective is the control and healing of the bullous skin and/or mucous lesions while minimizing, as much as possible, serious side-effects of treatment.

The treatment aims are as follows:

Healing of the bullous eruption and disappearance of the functional impairment associated with the disease;

Prevent/strictly limit the appearance of recurrences;

Improve the quality of life of the patients;

Limit common side-effects usually associated with long-term immunosuppressive or corticosteroid treatment.

\section{Professionals involved}

The initial management, diagnosis and treatment of extensive manifestations of the disease usually require hospitalization in a dermatology department.

This is continued until clinical control of the bullous eruption is achieved.

In limited forms of pemphigus, additional diagnostic examinations and clinical monitoring can be either performed in an inpatient or outpatient setting.

Overall management is coordinated by the dermatologist in liaison with the referring dermatologist, the general physician and other medical specialists and hospital doctors from the centre of reference and/or geographical area (if a reference centre exists in the particular country).

Specialists and health professionals involved are identical to those listed in the initial evaluation (see $\$ 2.2$ ). 
Exceptionally, the disease can occur during childhood, and children should be supported by a multidisciplinary team, jointly by a reference centre, a paediatric dermatology department or a paediatrician.

\section{Therapeutic management}

Mild pemphigus The following definition of mild pemphigus is recommended: $(4.9 \pm 0.3)$

- PF with involved body surface area (BSA) $<5 \%$.

- PV with involved body surface area (BSA) $<5 \%$ and limited oral lesions not impairing food intake or requiring analgesics.

- PDAI score $\leq 15 .^{23}$

\section{A. Mild pemphigus foliaceus}

Initial treatment of mild PF. Despite the fact that few of these treatments have been robustly evaluated in mild types of PF, the following initial therapies may be considered (Fig. 1):
Dapsone: start with 50 to $100 \mathrm{mg} /$ day, adjusted to clinical response up to $1.5 \mathrm{mg} / \mathrm{kg}$ bodyweight. Dapsone is usually combined with topical corticosteroids (classes III and IV) $(4.4 \pm 1.3) .{ }^{24}$ However, around $50 \%$ of patients who are started with dapsone without oral corticosteroids further relapse and secondarily need a systemic corticosteroid treatment. ${ }^{25}$ Additionally, dapsone failed to demonstrate a corticosteroid-sparing effect over corticosteroids alone in a randomized controlled study. ${ }^{26}$

Or

Topical corticosteroids: (class III, IV) alone if there are only very limited lesions $(4.3 \pm 1.4)$.

Or

Systemic corticosteroid therapy with prednisone $0.5-1.0 \mathrm{mg} /$ $\mathrm{kg} /$ day $(4.7 \pm 1.0)$.

Or

rituximab (two infusions of $1 \mathrm{~g}$ two weeks apart) alone, or associated with topical corticosteroids, ${ }^{27}$ or oral corticosteroids (prednisone $0.5 \mathrm{mg} / \mathrm{kg} /$ day) ${ }^{18}$ with a rapid decrease in order to stop corticosteroids after 3 to 4 months $(4.6 \pm 1.2)$.

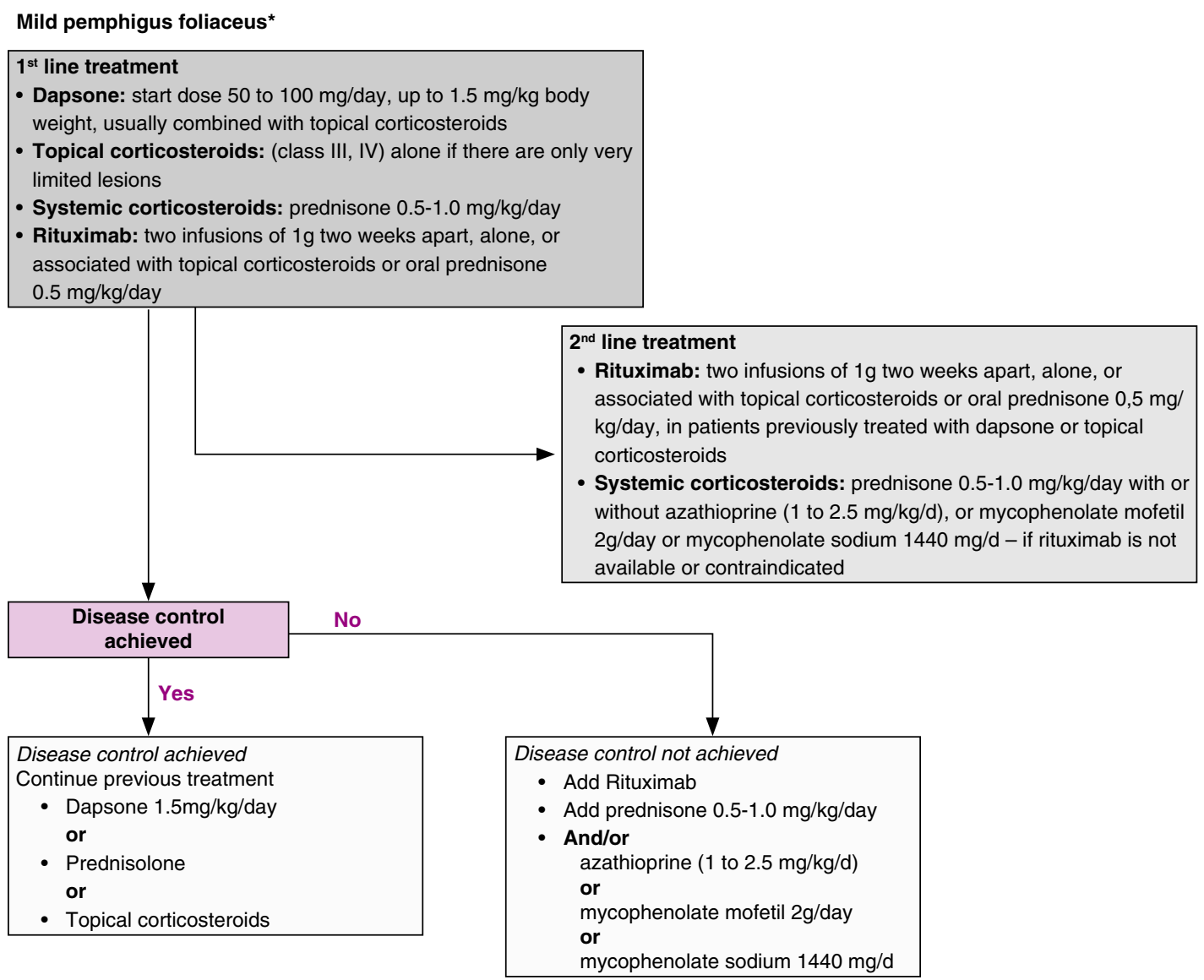

Figure 1 Treatment algorithm for mild pemphigus foliaceus. *involved body surface area $<5 \%$ and/or PDAl score $\leq 15$. 
Second line-The following second-line therapies of mild PF may be recommended:

In patients who have been initially treated with dapsone and/ or topical corticosteroids (without oral corticosteroids or rituximab) and have persistent active lesions and detection of antiDsg1 serum antibodies and significant impact on quality of life (ABQOL or DLQI scores):

- rituximab (two infusions of $1 \mathrm{~g}$ two weeks apart) alone or associated with oral corticosteroids (prednisone $0.5 \mathrm{mg} / \mathrm{kg} /$ day) with a rapid decrease in order to stop corticosteroids after 3 or 4 months $(4.7 \pm 0.6){ }^{16,28}$

Or ( if rituximab is contraindicated or not available)

Systemic corticosteroid therapy with prednisone $0.5-1.0 \mathrm{mg} /$ $\mathrm{kg} /$ day with or without azathioprine (1 to $2.5 \mathrm{mg} / \mathrm{kg} / \mathrm{day}$ ), or mycophenolate mofetil $2 \mathrm{~g} /$ day or mycophenolate sodium $1,440 \mathrm{mg} /$ day $(4.8 \pm 1.5) .^{29,30}$

In patients who have been initially treated with oral corticosteroid therapy alone and have persistent active lesions, add rituximab $(4.9 \pm 1.2)$.

\section{B. Mild pemphigus vulgaris}

Initial treatment of mild $P V$.

The following initial therapies of mild PV are recommended (Fig. 2):

Systemic corticosteroid therapy with prednisone $0.5-1.0 \mathrm{mg} /$ $\mathrm{kg} /$ day with or without azathioprine $(2.0 \mathrm{mg} / \mathrm{kg} /$ day $)$, or mycophenolate mofetil $2 \mathrm{~g} /$ day or mycophenolate sodium $1,440 \mathrm{mg} /$ day $(4.6 \pm 1.0)$.
Or

rituximab (two infusions of $1 \mathrm{~g}$ two weeks apart) alone, or associated with oral corticosteroids (prednisone $0.5 \mathrm{mg} / \mathrm{kg} /$ day) with a rapid decrease in order to stop corticosteroids after 3 or 4 months $(4.6 \pm 1.7){ }^{18,31,32}$

The following second-line therapies of mild PV are recommended:

In patients initially treated with prednisone/prednisolone $0.5-1.0 \mathrm{mg} / \mathrm{kg} /$ day alone who have persistent active lesions, and in patients with CS side-effect or contraindication to conventional immunosuppressant, and detection of anti-Dsg3 serum antibodies and significant impact on quality of life (ABQOL or DLQI scores): $(4.8 \pm 2.2)$.

- Addition of rituximab (two infusions of $1 \mathrm{~g}$ two weeks apart) with a rapid decrease of oral prednisolone in order to stop corticosteroids after 3 to 4 months $(4.8 \pm 1.0)$.

In patients initially treated with prednisone/prednisolone 0.5 $1.0 \mathrm{mg} / \mathrm{kg} /$ day plus rituximab, who have persistent active lesions, it is recommended to increase the dose of prednisone/ prednisolone up to $1 \mathrm{mg} / \mathrm{kg} /$ day $(4.6 \pm 0.7)$.

Moderate and severe types of pemphigus (PV and PF) The following definition of moderate and severe pemphigus is recommended: (4.8 \pm 0.5$)$ (Fig. 3 ).

- Multiple mucosal involvement of PV: oral, nasopharyngeal, conjunctival, genital

- Severe oral lesions or dysphagia with weight loss

- Significant pain

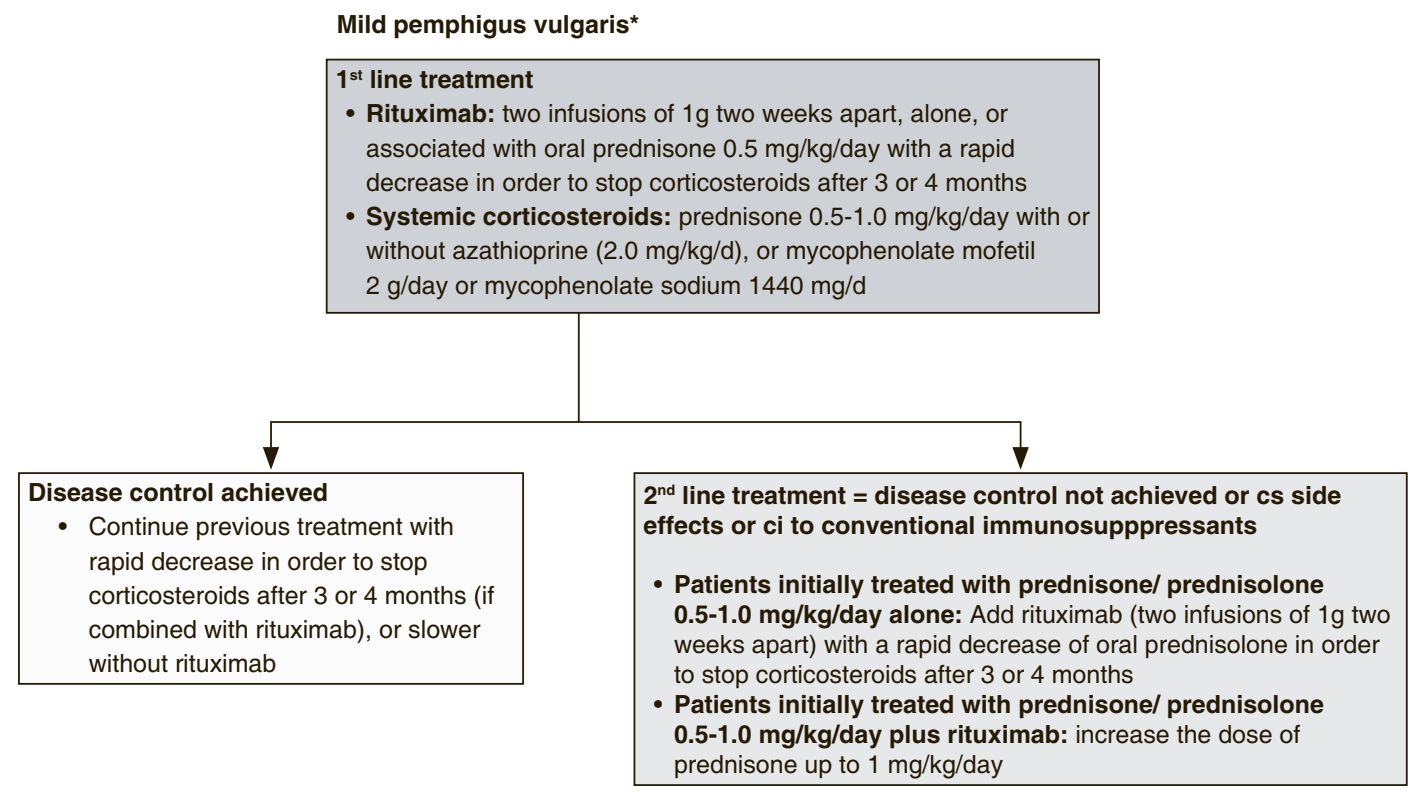

Figure 2 Treatment algorithm for mild pemphigus vulgaris. *involved body surface area $<5 \%$ and/or limited oral lesions not impairing food intake or requiring analgesics and/or PDAI score $\leq 15$. 
- And/or skin lesions $>5 \%$ BSA.

Moderate pemphigus: PDAI score $>15$ and $\leq 45$

Severe pemphigus: PDAI score $>45 .^{23}$

First-line treatment of moderate and severe types of pemphigus. Since there is no evidence in the literature that moderate and severe types of pemphigus must be treated in a different way, the proposals below refer to both moderate and severe types of the disease.

The following first-line therapies of moderate and severe pemphigus are recommended:

- Rituximab (two infusions of $1 \mathrm{~g}$ two weeks apart) associated with systemic corticosteroids (prednisone $1 \mathrm{mg} / \mathrm{kg} /$ day) with a progressive tapering in order to stop corticosteroids after 6 months $(4.9 \pm 0.4) .^{18,31,32}$

NB1: Rituximab can be administered as a monotherapy or associated with topical corticosteroids ${ }^{16,27}$ in patients with absolute contraindication to systemic corticosteroid therapy. $(4.7 \pm 0.9)$.

Or ( if rituximab is contraindicated or not available):

- Systemic corticosteroid therapy (oral prednisone 1 to $1.5 \mathrm{mg} / \mathrm{kg} /$ day) alone or associated with an immunosuppressive drug as corticosteroid-sparing agent (azathioprine 1 to $2.5 \mathrm{mg} / \mathrm{kg} /$ day or mycophenolate mofetil $2 \mathrm{~g} /$ day or mycophenolate sodium 1,440 mg/day), particularly in patients with an increased risk of severe corticosteroid side-effect related to an expected prolonged use of corticosteroids, or if there is no possibility to treat the patient with rituximab. ${ }^{1,29,30}$ Clinicians should be cautious for side-effects, relative and absolute contraindication of systemic corticosteroid therapy $(4.8 \pm 0.4)$.

In patients with neoplasia (paraneoplastic pemphigus or other type of pemphigus associated with cancer), it is recommended to consult with an oncologist, before the use of anti-CD20 monoclonal antibodies $(4.9 \pm 0.4)$.

Maintenance treatment after the initial cycle of rituximab. Patients' status 6 months after the first cycle of rituximab (month 6) - In patients who are in complete remission on/off therapy at month 6 , and initially presented with a severe pemphigus and/or still have a high rate of anti-Dsg antibodies at month 3, it may be considered to perform an infusion of $500 \mathrm{mg}$ or $1 \mathrm{~g}$ of rituximab at month $6 .^{28,33,34}$ The optimal dose (500 $\mathrm{mg}$ to $1 \mathrm{~g}$ ) has not been determined yet $(4.7 \pm 0.6)$.

\section{Moderate to severe PV/PF}

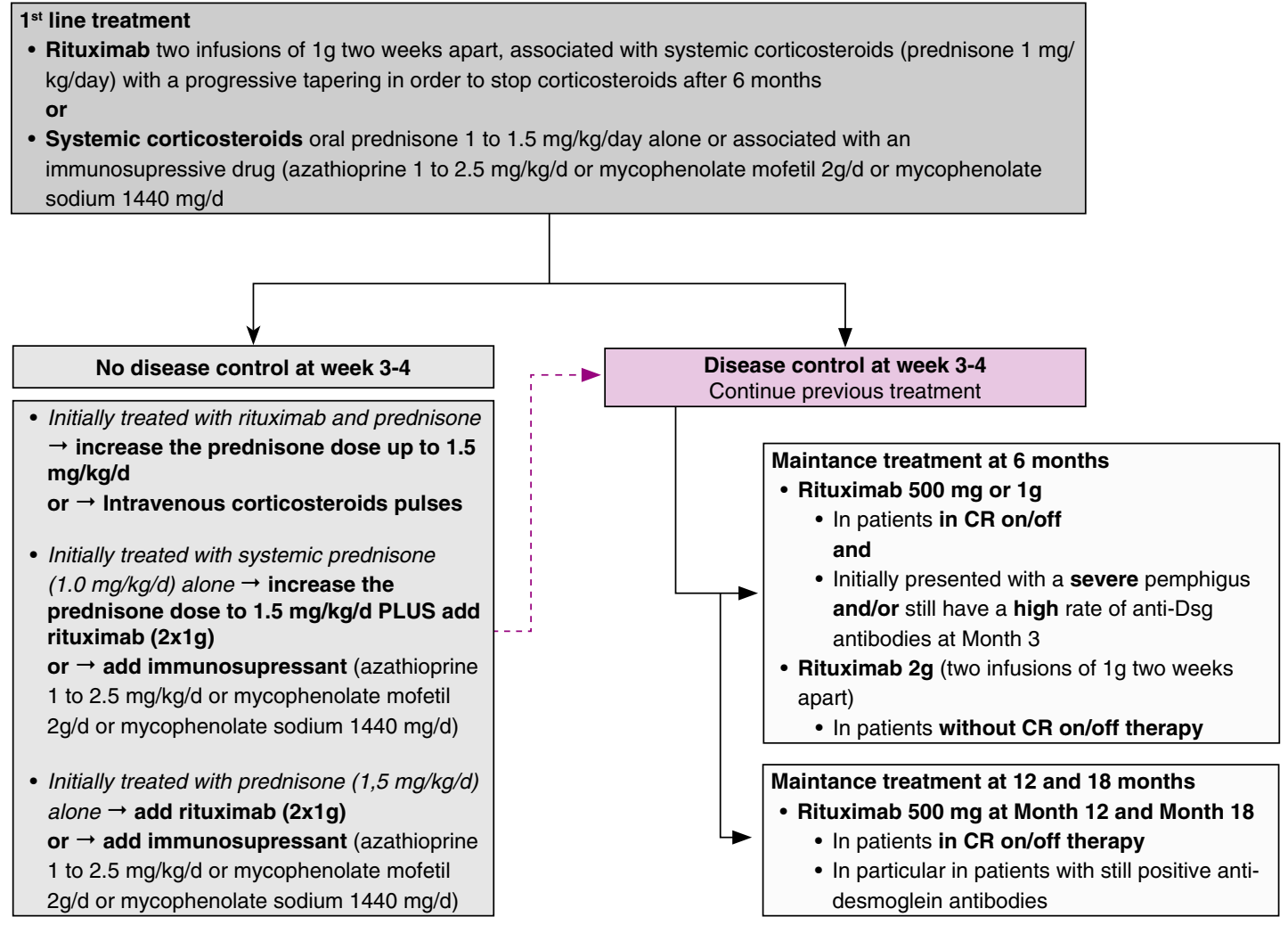

Figure 3 Treatment algorithm for moderate and severe pemphigus vulgaris and pemphigus foliaceus. CR, complete remission 
In patients without complete remission on/off therapy at month 6, it may be recommended to perform two infusions of $1 \mathrm{~g}$ two weeks apart $\left(2 \mathrm{~g}\right.$ in total) $(4.8 \pm 0.5){ }^{28,33}$

Months 12 and 18 after the first cycle (M12 and M18) - In patients in complete remission on/off therapy, it is recommended to perform one infusion of rituximab $(500 \mathrm{mg})$ at month 12 and another infusion of $500 \mathrm{mg}$ at month 18, in particular in patients with still positive anti-Dsg antibodies $(4.2 \pm 1.4) .^{34,35}$

The literature does not provide sufficient evidence to recommend systematic infusions of rituximab after month 18 in patients in complete remission. ${ }^{36}$ Monitoring of circulating antiDsg antibody ELISA values is necessary at least every six months $(4.7 \pm 1.2)$.

It may be considered to perform additional infusions of rituximab as maintenance therapy after month 18 in patients with a re-increase of anti-Dsg antibodies after their initial disappearance following the initial infusions of rituximab. $(4.6 \pm 1.1){ }^{34,35,36}$

Absence of initial disease control (after 3 to 4 weeks of treatment). In patients initially treated with rituximab and prednisone-It is recommended to increase the prednisone dose up to $1.5 \mathrm{mg} / \mathrm{kg} /$ day $(4.6 \pm 0.7)$.

Or

Intravenous corticosteroid pulses: methylprednisolone 0.5$1 \mathrm{~g} /$ day or dexamethasone $100 \mathrm{mg}$ /day over 3 consecutive days in initial intervals of $3-4$ weeks $(4.3 \pm 0.9){ }^{37}$

In patients initially treated with systemic corticosteroids alone (at an initial dose of prednisone, $1.0 \mathrm{mg} / \mathrm{kg} /$ day) _ It is recommended to increase the prednisone dose to $1.5 \mathrm{mg} / \mathrm{kg} / \mathrm{day}$ and add rituximab $(2 \times 1 \mathrm{~g})$, or if there is no possibility to treat the patient with rituximab, propose the addition of an immunosuppressant (azathioprine 1 to $2.5 \mathrm{mg} / \mathrm{kg} /$ day or mycophenolate mofetil $2 \mathrm{~g} /$ day or mycophenolate sodium $1,440 \mathrm{mg} /$ day) $(4.6 \pm 0.7)$.

In patients treated with systemic corticosteroids alone (at an initial dose of prednisone, $1.5 \mathrm{mg} / \mathrm{kg} /$ day) - If there is no possibility to treat the patient with rituximab, it is recommended to add a corticosteroid-sparing agent (azathioprine 1 to $2.5 \mathrm{mg} / \mathrm{kg} /$ day or mycophenolate mofetil $2 \mathrm{~g} /$ day or mycophenolate sodium $1,440 \mathrm{mg} /$ day). Cyclophosphamide is less frequently used due to its potentially severe side-effects $(4.9 \pm 0.3){ }^{1}$

In patients with severe/refractory pemphigus-Three other treatments may be recommended (in addition to rituximab, or if there is no response to rituximab treatment, or in addition to an immunosuppressant if there is no possibility to treat the patient with rituximab): $(4.8 \pm 2.3)$

- Intravenous immunoglobulins (IVIG) 2 g/kg/cycle (over 25 consecutive days every 4 weeks). ${ }^{38}$ Treatment can also be performed over several days to avoid headache and nausea. Aseptic meningitis is a rare but important side-effect of IVIG treatment which needs to be considered, in particular in patients who commonly experience episodes of migraine. Even though rare, complete IgA deficiency is a contraindication for IVIG treatment $(4.8 \pm 1.0)$.

- Intravenous corticosteroid pulses: methylprednisolone 0.5$1 \mathrm{~g} /$ day or dexamethasone $100 \mathrm{mg} /$ day over 3 consecutive days in initial intervals of 3-4 weeks $(4.2 \pm 1.5){ }^{37}$

- Immunoadsorption (minimum of 2 cycles over 3-4 consecutive days which are performed 4 weeks apart). Contraindications include severe systemic infections, severe cardiovascular diseases, hypersensitivity against components of the immunoadsorption column, treatment with angiotensin-converting enzyme inhibitors and extensive haemorrhagic diathesis $(4.3 \pm 1.3){ }^{39,40}$

Conventional immunosuppressive adjuvant Conventional adjuvants may be considered as first-line therapy when rituximab is not available, or not permitted as first line, or in patients with contraindications to rituximab.

The main immunosuppressants used as first-line treatments in pemphigus are azathioprine and mycophenolate mofetil ${ }^{41}$ $(4.8 \pm 1.2)$.

- Azathioprine (1-2.5 mg/kg/day). Start first week $50 \mathrm{mg} /$ day to detect idiosyncratic reactions (and in case stop immediately), and then raise to desired dose. Even though not predictive for idiosyncratic reactions, thiopurine methyl transferase (TMTP) activity should be monitored prior to treatment because the recommendations for azathioprine dosing vary based upon TPMT activity. ${ }^{41}$ In general, adults with pemphigus and high TPMT activity are treated with normal doses of azathioprine (up to $2.5 \mathrm{mg} / \mathrm{kg} / \mathrm{day}$ ), patients with intermediate or low TPMT activity should receive a lower maintenance dose (up to 0.5 to $1.5 \mathrm{mg} / \mathrm{kg}$ / day) depending on level of enzyme activity, and patients with lack of TPMT activity should not be treated with azathioprine.

- Mycophenolate mofetil (MMF) (2 g/day) or mycophenolic acid $(1,440 \mathrm{mg} /$ day). In case of MMF, consider to raise daily dose by 1 capsule (500 $\mathrm{mg}$ ) per week until the final dose of $2 \mathrm{~g} /$ day for better gastrointestinal tolerance. $^{29}$

- Methotrexate and cyclosporine are not recommended $(4.3 \pm 1.2)$.

If conventional immunosuppressants (which must be used as first line for regulatory reasons in some countries) fail, rituximab may be applied as second line.

- Cyclophosphamide (p.o. $50 \mathrm{mg} /$ day or i.v. $500-750 \mathrm{mg} /$ month) may be considered as third-line treatment in recalcitrant cases of pemphigus due its potentially severe side-effects. $(4.5 \pm 1.1)$. 


\section{Additional supportive treatment}

Intralesional injections of corticosteroids (triamcinolone acetonide) may be considered for isolated lesions of oral mucosa, lips and skin.

Topical adjuvant treatment with super-potent corticosteroids (clobetasol propionate or triamcinolone acetonide gel) directly to oral erosions may be considered in some patients in combination with systemic therapy.

The use of baths containing antiseptics such as chlorhexidine may be recommended in patients with extensive skin lesions.

It is recommended to cover erosive lesions with low adhesive wound dressings or local emollients, and compresses.

Analgesics (paracetamol, metamizol and opioids) are recommended if required.

Gels containing local anaesthetics may be recommended for application at the mucosal surfaces.

Proper dental care is recommended.

Extensive dental surgery, e.g. dental implants, is not recommended before the healing of oral lesions.

Nutritional management with the help of a dietician or a nutritionist is recommended if malnutrition is related to oral involvement or systemic corticosteroid therapy.

\section{Measures in prolonged corticosteroid therapy}

It is recommended to use vitamin D and calcium supplementation at initiation of glucocorticoid treatment to prevent osteoporosis $(4.8 \pm 0.8)$.

It is recommended to use bisphosphonates (i.e. alendronate, risedronate) in patients at risk (postmenopausal women, men $>50$ years, with pathological initial osteoporosis screening) to prevent osteoporosis $(4.9 \pm 0.4)$.

It may be considered to perform regular ophthalmologic evaluations during treatment with systemic corticosteroids $(4.8 \pm 0.7)$.

It may be recommended to use systemic antifungal, antiviral and antibiotic treatments only when clinically indicated $(4.9 \pm 0.2)$.

It may be considered to apply $\mathrm{H} 2$ blockers or proton pump inhibitors to prevent gastric/duodenal ulcers $(4.9 \pm 0.4)$. Based on insufficient evidence, the decision should be individualized to the patient, for example in case of additional treatment with non-steroidal anti-inflammatory drugs. ${ }^{42}$

It is recommended to offer a psychological support if required and to pay attention to depression, sometimes induced by corticosteroid treatment $(4.9 \pm 0.3)$.

It may be considered to apply physiotherapy if prolonged corticosteroid therapy is required $(4.6 \pm 0.7)$.

It may be considered to use antithrombotic prophylaxis in case of high risk of thrombosis $(4.5 \pm 0.3)$.

Vaccinations Adjuvant immunosuppressants and rituximab contraindicate the use of live vaccines.
It is recommended that patients receiving oral corticosteroids or immunosuppressive therapy are vaccinated against seasonal influenza, and pneumococci $(4.7 \pm 0.6)$. Other standard vaccinations (tetanus, diphtheria, pertussis, polio...) are recommended to be updated.

\section{Monitoring}

Pemphigus often shows a chronic (relapsing) course which requires close monitoring of clinical symptoms and of potential side-effects inherent to chronic immunosuppressive treatment. Thus, a multidisciplinary approach is commonly required.

\section{Objectives}

To evaluate the efficacy and safety of treatment.

To plan the gradual reduction of immunosuppressive treatment, and the duration of maintenance therapy or its discontinuation.

In clinical studies, it is recommended to use the definition of disease and outcome parameters as described by Murrell et al. $^{21,22}(4.9 \pm 0.9)$.

Approach to be maintained after consolidation phase It is recommended to taper the systemic corticosteroids over the next 4-6 months if treatment is associated with rituximab $(4.8 \pm 1.2) .^{18}$

The following tapering regimen may be recommended:

\begin{tabular}{lll}
\hline & Mild pemphigus & Moderate/severe pemphigus \\
\hline Month 1 & $0.5 \mathrm{mg} / \mathrm{kg}$ & $1 \mathrm{mg} / \mathrm{kg}$ \\
Month 2 & $0.3 \mathrm{mg} / \mathrm{kg}$ & $0.75 \mathrm{mg} / \mathrm{kg}$ \\
Month 3 & $0.2 \mathrm{mg} / \mathrm{kg}$ & $0.5 \mathrm{mg} / \mathrm{kg}$ \\
Month 4 & $\pm 0.1 \mathrm{mg} / \mathrm{kg}$ & $0.3 \mathrm{mg} / \mathrm{kg}$ \\
Month 5 & - & $0.2 \mathrm{mg} / \mathrm{kg}$ \\
Month 6 & - & $0.1 \mathrm{mg} / \mathrm{kg}$ \\
\hline
\end{tabular}

Beyond the last month of corticosteroid treatment $(0.1 \mathrm{mg} /$ $\mathrm{kg}$ /day), the attitude is not clearly defined. Three possibilities may be considered: i) stopping corticosteroid treatment after performing an ACTH test; ii) slow tapering (mg per mg every month or more slowly); iii) maintaining a minimal dose of corticosteroids (usually between 3 and $6 \mathrm{mg} /$ day), especially in patients with persistent significant levels of circulating anti-Dsg antibodies $(4.5 \pm 0.9)$.

If systemic corticosteroids are used without rituximab, it is recommended to taper the corticosteroids according to the clinical response $(4.9 \pm 0.2)$.

No clear consensus could be reached between participants on the best corticosteroid tapering regimen: it is proposed to decrease prednisone dose by $10 \%-25 \%$ every $2-3$ weeks until $15-25 \mathrm{mg} /$ day $(4.0 \pm 1.1)$; once at $15-25 \mathrm{mg} /$ day, no consensus again was reached on the best way of tapering prednisone from $1 \mathrm{mg}$ every 3 to 4 weeks to a more rapid tapering using $5 \mathrm{mg}$ steps. It may be recommended to taper the prednisolone dose 
even slower as the rate of anti-Dsg $1>50 \mathrm{IU} / \mathrm{mL}$ (situation where the risk of skin relapse is increased) $(4.2 \pm 1.2)$. The persistence of high levels of anti-Dsg1 antibodies by ELISA has a positive predictive value for skin relapses, while the persistence of anti-Dsg3 IgG does not necessarily predict a mucosal relapse (except if $>130 \mathrm{IU} / \mathrm{mL})(4.5 \pm 0.8) .{ }^{43}$

Treatment of relapse In patients initially treated with rituximab and systemic corticosteroids:

- If the relapse occurs during tapering of prednisone between month 0 and month 4 , it is recommended to re-increase oral corticosteroids (depending on the severity of relapse) $(4.8 \pm 0.4)$.

- If the relapse occurs during tapering of prednisone between month 4 and month 6 , it is recommended to perform an additional cycle of $2 \mathrm{~g}$ of rituximab. In this case, a maintenance infusion of rituximab will not be performed at month $6(4.3 \pm 1.4)$.

- If the relapse occurs after stopping prednisone (after the month 6 maintenance infusion of rituximab): few evidence based data are available from the literature in this particular situation; we thus recommend an evaluation by an expert in the field of pemphigus $(4.8 \pm 0.5)$.

In patients not initially treated with rituximab:

- If the relapse occurs during tapering of the corticosteroid, it is recommended to apply rituximab ( $1 \mathrm{~g}$ two weeks apart) $(4.9 \pm 0.4)$.

- In patients with contraindications to rituximab or when rituximab is not available, it is recommended to re-increase the corticosteroids dose and add azathioprine (1 to $2.5 \mathrm{mg} /$ $\mathrm{kg} /$ day), or mycophenolate mofetil (2 g/day) or mycophenolate sodium $(1,440 \mathrm{mg} /$ day $)$ until disease control is achieved, before tapering of systemic corticosteroids $(4.9 \pm 0.9)$.

\section{Scheduling and content of consultations}

Evaluation of the efficacy of treatment is primarily based on clinical symptoms.

The frequency of disease management (physical examination, additional examinations) must be adapted:

To the patient's clinical condition;

To the severity and disease course during treatment using clinical scores (ABSIS or PDAI);

To the therapeutics used (monitoring, tolerance, side-effects). Follow-up visits are recommended every 2-4 weeks until disease control is achieved. $(4.7 \pm 0.7)$. Then, follow-up visits are recommended every $4-8$ weeks until corticosteroids have been omitted $(4.5 \pm 0.8)$.

Thereafter, follow-up visits are recommended every 816 weeks until complete remission off therapy is achieved and serum anti-Dsg antibodies are normalized $(4.8 \pm 0.5)$.
Clinical examination The clinical follow-up is identical to that carried out during the initial assessment, and it should seek to clarify:

If the disease is clinically controlled (mucosal, mucocutaneous or cutaneous lesions);

IF adverse effects related to treatment are present or absent;

Diabetes mellitus, high blood pressure, cardiac insufficiency (corticosteroids);

Respiratory disorders, anaemia, hepatitis (dapsone, methotrexate);

Infections, notably respiratory, hepatitis, CMV reactivation (corticosteroids, immunosuppressants);

Mental disorders (corticosteroids);

Myopathy, osteoporosis, avascular bone necrosis, glaucoma, cataract (glucocorticoids);

Haematological abnormalities (leucopenia), (immunosuppressants).

Serological monitoring of disease activity It is recommended to determine the level of serum autoantibodies at the initiation of treatment, after 3 months and every 3 to 6 months based on the evolution, or in case of relapse by ELISA: anti-Dsg1 and/or Dsg3 IgG (4.5 \pm 0.8$)$. If ELISA is not available IIF microscopy utilizing monkey oesophagus or Dsg 1/3-expressing cells can beused $(4.7 \pm 0.7)$.

Overall, serum concentrations of IgG autoantibodies against Dsg1 and, to a lesser degree, Dsg3 correlate with the clinical activity of pemphigus and may thus help in therapeutic decision-making. Indeed, due to their poor specificity, anti-Dsg3 Abs should be used with caution for the management of pemphigus, since only high titres are good predictor for the occurrence of a relapse. ${ }^{34,43}$

Discontinuation of treatment

Discontinuation of treatment is primarily based on the clinical symptoms in combination with findings of Dsg ELISA and/or IIF microscopy $(4.9 \pm 0.4) .^{25,43}$

Discontinuation of systemic corticosteroids may be considered after performing an ACTH test in patients in complete remission on minimal therapy (prednisolone or equivalent at $\leq 10 \mathrm{mg} /$ day) with negative circulating anti-Dsg antibodies $(4.4 \pm 1.0)$.

It is recommended to omit the corticosteroid-sparing adjuvants 6-12 months after achieving complete remission on therapy $(4.6 \pm 1.0)$.

\section{Possible sequelae}

Pemphigus may cause sequelae due to the involvement of skin, conjunctivae, oral, pharyngeal, laryngeal, oesophageal, anogenital and anal mucosa but also due to side-effects of treatment, justifying request for recognition or help from departmental disability centres. 


\section{Information for patients}

It is recommended to inform patients and their families about the disease, its clinical course and prognosis, treatment, relapse signs, possible adverse events associated with treatment $(5.0 \pm 0.0)$.

Written information is available by the EADV webpage and the patient support groups.

It is recommended to inform patients about patient support groups for pemphigus (see list below) $(4.9 \pm 1.0)$.

The purpose of these associations is to promote knowledge about the disease, provide comfort and share the experience of patients regarding daily life, and to provide information dissemination. It may contribute to a better overall management of the disease by promoting cooperation between patients, patient associations and health professionals. Patients are also informed about referral centres.

It may be recommended to alert patients about potential trigger factors such as certain drugs, operations, infections, radiation, and sun exposure $(4.8 \pm 0.5)$.

It is not recommended to propose dietetic restrictions $(4.3 \pm 1.3)$.

\section{List of pemphigus support groups}

International Pemphigus and Pemphigoid foundation: www. pemphigus.org

Association Pemphigus Pemphigoïde-France: www.pemphi gus.asso.fr

Pemfriends: www.pemfriends.co.uk

Pemphigus und Pemphigoid Selbsthilfegruppe e. V.: www.pe mphigus-pemphigoid-selbsthilfe.de

Associazione Nazionale Pemfigo/Pemfigoide Italy: www.pemf igo.org

Netwerk Nederland Pemphigus en Pemfigoïd: www.pemphi gus.nl

Pemfigus Hastaları Yardımlaşma ve Dayanışma Derneği: www.pemfigus.org.tr

\section{References}

1 Schmidt E, Kasperkiewicz M, Joly P. Pemphigus. Lancet Lond Engl 2019; 394: 882-894.

2 Joly P, Litrowski N. Pemphigus group (vulgaris, vegetans, foliaceus, herpetiformis, brasiliensis). Clin Dermatol. 2011; 29: 432-436.

3 Kneisel A, Hertl M. Autoimmune bullous skin diseases. Part 1: Clinical manifestations. J Dtsch Dermatol Ges J Ger Soc Dermatol JDDG 2011; 9: 844-856; quiz 857.

4 Kneisel A, Hertl M. Autoimmune bullous skin diseases. Part 2: diagnosis and therapy. J Dtsch Dermatol Ges J Ger Soc Dermatol JDDG 2011; 9: 927947.

5 Kasperkiewicz M, Ellebrecht CT, Takahashi H et al. Pemphigus. Nat Rev Dis Primer 2017; 3: 17026.

6 Amagai M, Klaus-Kovtun V, Stanley JR. Autoantibodies against a novel epithelial cadherin in pemphigus vulgaris, a disease of cell adhesion. Cell 1991; 67: 869-877.

7 Jelti L, Cordel N, Gillibert A et al. Incidence and mortality of pemphigus in France. J Invest Dermatol. 2019; 139: 469-473.
8 Hübner F, Recke A, Zillikens D, Linder R, Schmidt E. Prevalence and age distribution of pemphigus and pemphigoid diseases in germany. J Invest Dermatol. 2016; 136: 2495-2498.

9 Harman KE, Seed PT, Gratian MJ, Bhogal BS, Challacombe SJ, Black MM. The severity of cutaneous and oral pemphigus is related to desmoglein 1 and 3 antibody levels. Br J Dermatol. 2001; 144: 775-780.

10 Schmidt E, Dähnrich C, Rosemann A et al. Novel ELISA systems for antibodies to desmoglein 1 and 3: correlation of disease activity with serum autoantibody levels in individual pemphigus patients. Exp Dermatol 2010; 19: 458-463.

11 Martin LK, Werth V, Villanueva E, Segall J, Murrell DF. Interventions for pemphigus vulgaris and pemphigus foliaceus. Cochrane Database Syst Rev 2009; 1: CD006263.

12 Martin LK, Werth VP, Villaneuva EV, Murrell DF. A systematic review of randomized controlled trials for pemphigus vulgaris and pemphigus foliaceus. J Am Acad Dermatol 2011; 64: 903-908.

13 Hertl M, Jedlickova H, Karpati S et al. Pemphigus. S2 Guideline for diagnosis and treatment-guided by the European Dermatology Forum (EDF) in cooperation with the European Academy of Dermatology and Venereology (EADV). J Eur Acad Dermatol Venereol JEADV 2015; 29 :405-414.

14 Tavakolpour S, Mahmoudi H, Balighi K, Abedini R, Daneshpazhooh M. Sixteen-year history of rituximab therapy for 1085 pemphigus vulgaris patients: A systematic review. Int Immunopharmacol 2018; 54: 131-138.

15 Schmidt E, Goebeler M Zillikens D. Rituximab in severe pemphigus. Ann N Y Acad Sci 2009; 1173: 683-691.

16 Joly P, Mouquet H, Roujeau J-C et al. A single cycle of rituximab for the treatment of severe pemphigus. N Engl J Med 2007; 357: 545-552.

17 Ahmed AR, Spigelman Z, Cavacini LA, Posner MR. Treatment of pemphigus vulgaris with rituximab and intravenous immune globulin. $N$ Engl J Med 2006; 355: 1772-1779.

18 Joly P, Maho-Vaillant M, Prost-Squarcioni C et al. First-line rituximab combined with short-term prednisone versus prednisone alone for the treatment of pemphigus (Ritux 3): a prospective, multicentre, parallelgroup, open-label randomised trial. Lancet Lond Engl 2017; 389: 20312040.

19 Sebaratnam DF, Hanna AM, Chee S et al. Development of a quality-oflife instrument for autoimmune bullous disease: the Autoimmune Bullous Disease Quality of Life questionnaire. JAMA Dermatol 2013; 149: 1186-1191.

20 Tjokrowidjaja A, Daniel BS, Frew JW et al. The development and validation of the treatment of autoimmune bullous disease quality of life questionnaire, a tool to measure the quality of life impacts of treatments used in patients with autoimmune blistering disease. Br J Dermatol 2013; 169: 1000-1006.

21 Murrell DF, Dick S, Ahmed AR et al. Consensus statement on definitions of disease, end points, and therapeutic response for pemphigus. J Am Acad Dermatol 2008; 58: 1043-1046.

22 Hébert V, Boulard $\mathrm{C}$, Houivet $\mathrm{E}$ et al. Large international validation of ABSIS and PDAI pemphigus severity scores. J Invest Dermatol. 2019; 139: 31-37.

23 Boulard C, Duvert Lehembre S, Picard-Dahan C et al. Calculation of cutoff values based on the Autoimmune Bullous Skin Disorder Intensity Score (ABSIS) and Pemphigus Disease Area Index (PDAI) pemphigus scoring systems for defining moderate, significant and extensive types of pemphigus. Br J Dermatol 2016; 175:142-149.

24 Basset N, Guillot B, Michel B, Meynadier J Guilhou JJ. Dapsone as initial treatment in superficial pemphigus. Report of nine cases. Arch Dermatol 1987; 123: 783-785.

25 Almugairen N, Hospital V, Bedane C et al. Assessment of the rate of long-term complete remission off therapy in patients with pemphigus treated with different regimens including medium- and high-dose corticosteroids. J Am Acad Dermatol 2013; 69: 583-588.

26 Werth VP, Fivenson D, Pandya AG et al. Multicenter randomized, double-blind, placebo-controlled, clinical trial of dapsone as a 
glucocorticoid-sparing agent in maintenance-phase pemphigus vulgaris. Arch Dermatol 2008; 144: 25-32.

27 Ingen-Housz-Oro S, Valeyrie-Allanore L, Cosnes A et al. First-line treatment of pemphigus vulgaris with a combination of rituximab and highpotency topical corticosteroids. JAMA Dermatol 2015; 151: 200-203.

28 Cianchini G, Lupi F, Masini C, Corona R, Puddu P, De Pità O. Therapy with rituximab for autoimmune pemphigus: results from a single-center observational study on 42 cases with long-term follow-up. J Am Acad Dermatol 2012; 67: 617-622.

29 Beissert S, Mimouni D, Kanwar AJ, Solomons N, Kalia V, Anhalt GJ. Treating pemphigus vulgaris with prednisone and mycophenolate mofetil: a multicenter, randomized, placebo-controlled trial. J Invest Dermatol 2010; 130: 2041-2048.

30 Beissert S, Werfel T, Frieling U et al. A comparison of oral methylprednisolone plus azathioprine or mycophenolate mofetil for the treatment of pemphigus. Arch Dermatol 2006; 142: 1447-1454.

31 Hébert V, Vermeulin T, Tanguy L et al. Comparison of real costs in the French healthcare system in newly diagnosed patients with pemphigus for first-line treatment with rituximab vs. standard corticosteroid regimen: data from a national multicentre trial. Br J Dermatol. 2019; 183: 121-127. https://doi.org/10.1111/bjd.18563. Epub ahead of print.

32 Chen DM, Odueyungbo A, Csinady E et al. Rituximab is an effective treatment in patients with pemphigus vulgaris and demonstrates a steroid-sparing effect. Br J Dermatol. 2019; 182: 1111-1119. https://doi.org/ 10.1111/bjd.18482. Epub ahead of print.

33 Jelti L, Prost-Squarcioni C, Ingen-Housz-Oro S et al. Update of the French recommendations for the management of pemphigus. Ann Dermatol Venereol 2019; 146: 279-286.

34 Mignard C, Maho-Vaillant M, Golinski ML et al. Factors associated with short-term relapse in patients with pemphigus who receive rituximab as first-line therapy: A post hoc analysis of a randomized clinical trial. JAMA Dermatol 2020; 156: 1-8.

35 Colliou N, Picard D, Caillot F et al. Long-term remissions of severe pemphigus after rituximab therapy are associated with prolonged failure of desmoglein B cell response. Sci Transl Med 2013; 5: 175 ra30.

36 Sanchez J, Ingen-Housz-Oro S, Chosidow O, Antonicelli F Bernard P. Rituximab as Single Long-term Maintenance Therapy in Patients With Difficult-to-Treat Pemphigus. JAMA Dermatol 2018; 01: 363-365.

37 Werth VP. Treatment of pemphigus vulgaris with brief, high-dose intravenous glucocorticoids. Arch Dermatol 1996; 132: 1435-1439.

38 Amagai M, Ikeda S, Shimizu H et al. A randomized double-blind trial of intravenous immunoglobulin for pemphigus. J Am Acad Dermatol 2009; 60: 595-603.

39 Behzad M, Möbs C, Kneisel A et al. Combined treatment with immunoadsorption and rituximab leads to fast and prolonged clinical remission in difficult-to-treat pemphigus vulgaris. Br J Dermatol 2012; 166: 844-852.

40 Kasperkiewicz M, Shimanovich I, Meier M et al. Treatment of severe pemphigus with a combination of immunoadsorption, rituximab, pulsed dexamethasone and azathioprine/mycophenolate mofetil: a pilot study of 23 patients. Br J Dermatol 2012; 166: 154-160.

41 Meggitt SJ, Anstey AV, Mohd Mustapa MF, Reynolds NJ Wakelin S. British Association of Dermatologists' guidelines for the safe and effective prescribing of azathioprine 2011. Br J Dermatol 2011; 165: 711-734.

42 Guslandi M. Steroid ulcers: Any news? World J Gastrointest Pharmacol Ther 2013; 4: 39-40.

43 Abasq C, Mouquet H, Gilbert D et al. ELISA testing of anti-desmoglein 1 and 3 antibodies in the management of pemphigus. Arch Dermatol. 2009; 145:529-535. 